\title{
POSTER
}

\section{Tuméfaction palatine révélatrice d'un adénome pleiomorphe}

\section{Royer G', llie $\mathbf{M}^{2}$, Afota F', Santini J3 , Savoldelli $\mathrm{C}^{4}$}

1 - Interne DESCO

2 - MD, PhD, Laboratory of Clinical and Experimental Pathology, Pasteur Hospital, Nice, France

3 - PU-PH, IUFC, Nice

4 - MCU-PH, IUFC, Nice

Un patient de 60 ans s'est présenté en consultation pour une tuméfaction palatine évoluant depuis 5 mois. L'aspect de cette tuméfaction violacée sans bourgeonnement évoquait une origine salivaire sans conclure au caractère bénin ou malin. Le scanner réalisé mettait en évidence un refoulement de la corticale palatine sans lyse osseuse. L'exérèse de la lésion a été réalisée. L'examen anatomopathologique a conclu à un adénome pléomorphe. L'adénome pléomorphe est une tumeur bénigne aussi appelée tumeur mixte due à l'association au sein du même tissu d'une composante épithéliale et d'une composante mésenchymateuse. Elle représente la plus fréquente des tumeurs des glandes salivaires (45 à $65 \%$ ) (1). Elle siège le plus souvent dans les glandes principales, essentiellement la parotide, mais atteint également les glandes salivaires accessoires. Elle touche dans ce cas là le palais dur ou mou ( $5 \%$ des cas) ou la lèvre supérieure, mais peut se développer sur toute la muqueuse buccale.

\section{Références}

(1) Pinkston JA, et al. Otolaryngol Head Neck Surg 1999;120:834-40. 